\title{
Giant Cell Tumor of Bone: A rare uncommon manifestation implicated in a new mosaic of desorders?
}

Eliandro de Souza Freitas ${ }^{1}$, Francisco Amadis Batista Ferreira ${ }^{2}$, Brendo Vinícius Rodrigues Louredo $^{3}$, Milena Gomes Melo Leite ${ }^{1}$, José da Cruz Luna Neto ${ }^{4}$, Fábio Arruda Bindá ${ }^{5}$, Caroline Alfaia Silva ${ }^{6}$, and Jeconias Câmara ${ }^{6}$

${ }^{1}$ Universidade do Estado do Amazonas Escola Superior de Ciencias da Saude ${ }^{2}$ Oral and Maxillofacial Surgery Section Adriano Jorge Hospital Foundation (FHAJ) Manaus

${ }^{3}$ Universidade Estadual de Campinas Faculdade de Odontologia de Piracicaba ${ }^{4}$ Centro Universitario do Norte

${ }^{5}$ Department of Head and Neck Surgery Amazonas State Oncology Control Center Foundation (FCECON) Planalto

${ }^{6}$ Universidade Federal do Amazonas

November 13, 2021

\begin{abstract}
Giant Cell Tumor of Bone is an uncommon aggressive benign neoplasm that frequently affecting the epiphysis of long bones especially around the knee. This is a singular rare case of GCTB in the right maxillary sinus implicated in a process coincidentally or casually with another type of lesion primarily developed.
\end{abstract}




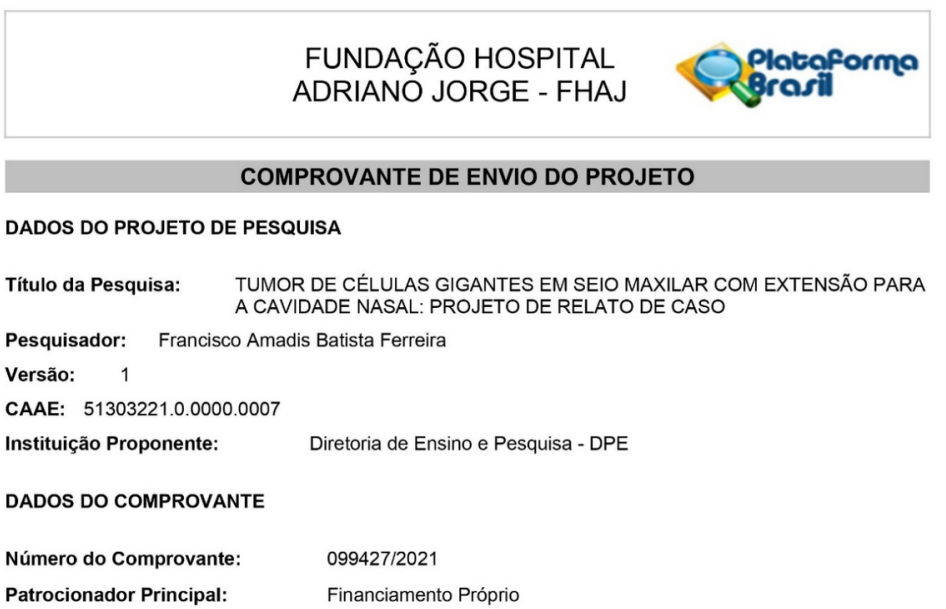

Informamos que o projeto TUMOR DE CÉLULAS GIGANTES EM SEIO MAXILAR COM EXTENSÃO PARA A CAVIDADE NASAL: PROJETO DE RELATO DE CASO que tem como pesquisador responsável Francisco Amadis Batista Ferreira, foi recebido para análise ética no CEP Fundação Hospital Adriano Jorge - FHAJ em 31/08/2021 às 10:49.

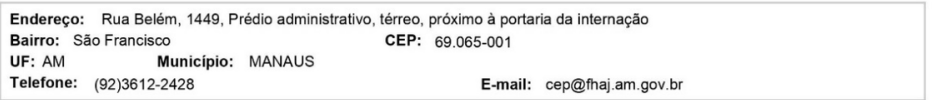

\section{INTRODUCTION}

Giant Cell Tumor of Bone (GCTB) is uncommon benign lesion, but locally aggressive that most frequently affecting the epiphysis of long bones especially around the knee [1]. The epiphyseal region is the common site for GCTB development, however, other frequent regions may occur as the distal femur, proximal tibia, distal radius and sacrum, rarely reported in the bones of the head, some reports of bones developing from endochondral ossification, such as sphenoid, ethmoid and temporal bone, are almost exclusively involved [3]. In the gnathic bones, the clinical appearance may vary, causing since dental root resorption to bone destruction, pain, pathologic fractures and metastatic spread [4]

It has a clinically aggressive behavior, with a fast growth, sometimes in weeks, however has few symptoms, leading to thinning and rupture of bone cortical, invasion of adjacent soft tissues, but not invading or 
ulcerating skin and subcutaneous tissue [5]. Albeit the GCTB belongs to the category of benign tumors, some studies prefer to characterize it as having "intermediate malignant potential" due to osteolytic properties causing invasion and destruction of adjacent structures [5].

The treatment for GCTB also may vary, depending on the aggressiveness of the tumor. Surgical resection, Embolisation, Bisphosphonates, Anti-RANKL therapy, Chemotherapy and Radiotherapy can be used ${ }^{4}$. Nowadays, the use of Denosumab has gained prominence as an adjuvant therapy by the inhibitory effects on RANKL, promoting significant reduction in tumor size, restoration of bone stock, and significant pain reduction in treated patients [6]

A comprehensive study of differential diagnosis should include tumors with similar histological appearance as well as benign or malign epithelial neoplasms with common chronic inflammatory conditions in the gnathic bones, nasal cavity and paranasal sinuses, such as brown tumor of hyperparathyroidism, malignant fibrous histiocytoma, giant cell central granuloma and chronic inflammatory masses from nasal cavity $[7,8]$

In this context, we aimed to present a singular rare clinical case of GCTB in the right maxillary sinus extending to the nasal cavity and skull base, implicated in a process coincidentally or casually with another type of lesion primarily developed.

\section{CASE REPORT}

All procedures performed in this study were in accordance with the ethical standards of the institutional research committee of Adriano Jorge Hospital Foundation (document number 51303221.0.0000.0007) and with the 1964 Helsinki declaration and its later amendments or comparable ethical standards. The patient provided his written consent, and no identifier information is included in this article.

A 33-year-old male patient arrived at the oral and maxillofacial surgery service complaining of painful swelling, obstruction, and bleeding in nasal cavity about 6 months. The physical examination showed a firm mass that obliterated and externalized through the right nasal cavity with intact skin and no ulceration. (figure 1a ). The patient reported medical history of incisional biopsy 4 years ago, in the same region, of a tumor with histopathological diagnosis of the "Suggestive of Nasal Paraganglioma", however the immunohistochemical study was negative for this tumor and compatible with polypoid tissue presenting an exuberant inflammatory process with numerous multinucleated giant cells in between and with positive markers for AE1/AE3 and CD68(table) (figure 2a, 2b, 2b). Due to the distance, difficult mobility and financial issues to get to the referral hospital, the patient did not return to subsequent appointments, not undergoing the proper treatment for the first lesion. During the Computed Tomography evaluate was observed a hyperdense mass occupying the entire maxillary sinus with no signs of aggressive resorption, perforation or tissue spread. In the Nuclear Magnetic Resonance (NMR) evaluate was showed hypersignal mass occupying the right maxillary sinus, obliterating the right nasal cavity and extending to skull base. (figure 1b,1c ). A bone window was performed in the right maxillary sinus through the Weber-Ferguson incision and an excisional biopsy from the mass was made, the osteotomy was reduced and fixated with plaques and screws of the $2.0 \mathrm{~mm}$ system and the surgical specimen was sent to anatomopathological analysis. (figure 1d,1e ). The patient has not undergone for any adjuvant therapy, and has been followed for 3 years with no signs of recurrence or complication. Grossly, the surgical specimen had a dark brown-to-reddish appearance that was friable in texture (figure 1f ). Microscopical investigation revealed benign neoplasm characterized by a highly cellular tumor dominated by large numbers of osteoclast-like giant cells. The multinucleated giant cells showed variable number nuclei, some with $>20$ per cell, with nuclei arranged disorganized in most giant cells. Otherwise, some areas showed multinucleated giant cells with a ring of nuclei surrounding a central homogeneous cytoplasm similar to Touton giant cells. The cytoplasm surrounded by the nuclei was both amphophilic and eosinophilic, while the cytoplasm near the periphery of the cell was pale and foamy in appearance. The mononuclear cells exhibited a variety of morphological appearances, including round to oval cells and spindled cells in a fibrotic background in most areas. In some fields, it was evident the presence of a large proliferation of xanthoma cells PAS-positives. Proliferation of vascular spaces and hemorrhage completed the analyzed fields. Atypical mitotic figures and necrosis were not observed. The features of 
malignancy like significant nuclear atypia or necrosis were not seen. Then, the diagnosis was well consistent with GCTB (figure 3a,3b,3c,3d,3e,3f ).

\section{DISCUSSION}

The WHO classification of tumors define the GCTB as a primary intra-medullary bone tumor composed of mononucleated cells and osteoclast-like multinucleated giant cells and presenting as a locally aggressive lesion with unpredictable behavior [7]. It is believed that the stroma represents the active portion of the tumor cells, capable to secreting differentiation factors that recruit monocytes, promoting their fusion and forming the third cell type in CGTB, the osteoclastic-like multinucleated giant cells [7].

For any benign or malignant pathological process, the stroma plays a fundamental role in its development, composed of proteoglycans, glycoproteins and water. In the GCTB, cytokines and differential factors including MCP1, ODF and M-CSF are present, suggesting that these molecules are essential to genesis of osteoclastic-like multinucleated giant cells [8]. However, several chronic inflammatory lesions with giant cell configuration may have similar histopathologic features, principally in its beginning. In our case, the primarily lesion developed 4 years ago, have shown already the positivity of CD68 marker and low activity of multinucleated giant cells in between, however, we believed that the size of the incisional biopsy performed previously could have compromised the global assessment of the lesion, omitting important characteristics of its development. Furthermore, there is a possibility that this tumor may represent a giant cell tumor of the bone arising ectopically placed in this region. Although we cannot exclude this hypothesis we do not have support to affirm this.

The WHO describe the histological appearance of GCTB as a highly cellular lesion typically dominated by large numbers of non-neoplastic osteoclast-like giant cells, between which mononuclear cells are embedded. The giant cells have a variable number of nuclei, some with $>50$ per cell. Mononuclear cells in GCTB exhibit a variety of morphological appearances, including round to oval cells in a fibrotic background and spindle cells associated with fibrous matrix. The presence of atypical mitotic figures should raise suspicion for malignancy. In the present case, it was observed this large number of multinucleated giant cells in all analyzed fields, some with $>20$ nuclei per cell. Also, the cytoplasm of the mononuclear cells was pattern epithelioid with nuclei similar to seen in multinucleated giant cells. The stroma was fibrous with spindle cells associated. However, atypical mitotic figures were not evident in this case. Furthermore, some areas contain hemorrhage focus and large number of xanthoma cells having abundant cytoplasm with fine vacuoles, which was also reported by other previous studies $[1,4,5,9]$.

Occasional Touton giant cells were noted in this case. Kumar et al. [10] analyzed 68 cases of GCTB of the tendon sheath. These 68 cases, osteoclastic-like giant cells were uniformly seen in all cases, while only nine $(13.2 \%)$ cases in addition showed Touton-type giant cells, which confirms that this finding is not a common feature in these tumors [10]. According Amary F. et al. the anti-histone H3.3 G34W Rabbit monoclonal antibody (clone RM263) is highly specific and sensitive for tumors and a powerful immunohistochemical marker for GCTB, improving the final diagnosis and biopsy specimens, allowing also, distinguishing GCTB from its mimics, like giant cell granulomas of the jaw, cherubism, brown tumors of hyperparathyroidism and foreign body-type giant cell reaction [11].

The management treatment for GCTB tumors depends on their location and radiological features. Surgical resection is the universal standard for treatment of GCTB [12]. Currently, the Denosumab is used as an adjuvant for causing suppression of osteoclastic lineage cells and preventing bone destruction [12]. Nevertheless, studies comparing different treatment methods with Denosumab has shown an increase rate of recurrence (20\% to $100 \%$ ) using curettage with preoperative denosumab comparing to curettage-alone group (0\% to 50\%) [13]. This different result, related with long-term therapeutic effect of denosumab, may cause dramatic changes in the tumor's histologic appearance, not eradicating mutational status of H3F3A and H3F3B, however careful evaluation is necessary for its use [14].

In summary, the association of the GCTB and the previous Polypoid mass was exceptionally uncommon and extremely rare. In our patient the first lesion occurred 4 years ago, with no signs of malignity or complication 
such as metastasis or spread and just incisional biopsy was made. The second lesion (GCTB) appeared as a silent event, gradually led to the development of a slow-growing lesion that was difficult to diagnose until it externalized through the right nasal cavity and is perceived by the patient. Although these conditions may occur coincidentally, rather than causally, we had to consider the possibility that these two entities were associated in our patient; we agree that possibly there was a relationship between the activity of the remaining polypoid tissue in the nasal cavity and the GCTB in all maxillary sinus. We do not have support to affirm that the first lesion become GCTB, certainly more control studies are needed for this.

To the best of our knowledge, there is only this report in the literature that describes a case with both GCTB and Polypoid tissue developed in the same location and in the head and neck region. Thus, we suggest to reach the diagnosis of GCTB and Polypoid tissue the combined use of Computed Tomography, Nuclear Magnetic Resonance, Histopathology, Immunohistochemistry, Blood Complementary Test, Bone Scintigraphy and Angiography depending on location, aggressiveness and size of lesion. Due to the fact that the patient is located in another city and does not have many financial conditions, we were not able to investigate in detail the possibly association of injuries. In addition, the public hospital where the clinical case was carried out lacks the resources to offer all these tests.

\section{CONCLUSION}

Therefore, the association between these lesions in the same location may be as coincidentally as a new process that requires further studies and still remains unknown, possibly implicated in a new model of clinical, microscopic and genetic disorders. Thus, will we be able to say in the future that masses from chronic inflammatory tissues origin, may have the potential to transform into GCTB?

\section{Authors' contributions:}

1. Author 1: conception and drafting of the manuscript;

2. Author 2: oral and maxillofacial surgeon of the case, conception and design of the manuscript;

3. Author 3: conception and design of the manuscript, pathology section;

4. Author 4: conception and design of the manuscript;

5. Author 5: conception and design of the manuscript;

6. Author 6: head and neck surgeon of the case;

7. Author 7: resident of oral and maxillofacial pathology

8. Author 8: oral and maxillofacial pathologist of the case

\section{REFERENCES}

1. Montgomery C, Couch C, Emory CL, Nicholas R. Giant Cell Tumor of Bone: Review of Current Literature, Evaluation, and Treatment Options. J Knee Surg. 2019;32(4):331-336.

2. Rosa D, Dias RB, Salvador JC, Borges A. Recurrent giant cell tumour of the maxillary sinus and pterygoid process treated with denosumab. BMJ Case Rep. 2018;2018:1-6.

3. Mohaidat ZM, Al-jamal HZ, Bany-Khalaf AM, Radaideh AM, Audat ZA. Giant cell tumor of bone: Unusual features of a rare tumor. Rare Tumors. 2019;11. doi:10.1177/2036361319878894

4. Sobti A, Agrawal P, Agarwala S, Agarwal M. Giant cell tumor of bone - An overview. Arch Bone Jt Surg. 2016;4(1):2-9.

5. di Carlo FS, Whyte MP \& Gianfrancesco F. The two faces of giant cell tumor of bone. Cancer Letters. 2020;489:1-8.

6. Luengo-Alonso G, Mellado-Romero M, Shemesh S, Ramos-Pascua L, Pretell-Mazzini J. Denosumab treatment for giant-cell tumor of bone: a systematic review of the literature. Archives of orthopaedic and trauma surgery. 2019;139(10):1339-1349.

7. Board WC of TE. Soft Tissue and Bone Tumours. 5th ed. Lyon (France): International Agency for Research on Cancer; 2020. 
8. Wülling, M., Engels, C., Jesse, N., Werner, M., Delling, G., \& Kaiser, E. The nature of giant cell tumor of bone. Journal of cancer research and clinical oncology. 2001;127(8):467-474.

9. Tuluc M., Zhang X, \& Inniss, S. Giant cell tumor of the nasal cavity: case report. European archives of oto-rhino-laryngology. 2007;264(2):205-208.

10. Kumar R, Bharani V, Gupta N, et al. Giant cell tumour of tendon sheath: A 10-year study from a tertiary care centre. Cytopathology. 2018;29(3):288-293.

11. Amary F, Berisha F, Ye H, Gupta M, Gutteridge A, et al. H3F3A (Histone 3.3) G34W immunohistochemistry: a reliable marker defining benign and malignant giant cell tumor of bone. The American journal of surgical pathology. 2017;41(8):1059.

12. Errani C, Tsukamoto S, Leone G, Righi A, Akahane M. et al. Denosumab may increase the risk of local recurrence in patients with giant-cell tumor of bone treated with curettage. JBJS. 2018; 100(6):496-504.

13. Tsukamoto S, Tanaka Y, Mavrogenis A. F, Kido A, Kawaguchi M et al. Is treatment with denosumab associated with local recurrence in patients with giant cell tumor of bone treated with curettage? A systematic review. A Publication of The Association of Bone and Joint Surgeons@| CORRß. 2020;478(5):1076-1085.

14. Kato I, Furuya M, Matsuo K, Kawabata Y, Tanaka R. et al. Giant cell tumours of bone treated with denosumab: histological, immunohistochemical and H3F3A mutation analyses. Histopathology. 2018;72(6):914922 .

\section{FIGURE LEGENDS}

\section{FIGURE 1:}

A) Clinical appearance of the tumor, externalizing through right nostril.

B) NMR showing a hypersignal mass occupying the right maxillary sinus, obliterating the right nasal cavity and extending to skull base.

C) Macroscopy appearance of the tumor.

D) Computed Tomography showing a hyperdense mass occupying the entire right maxillary sinus.

E) Weber-Ferguson incision to perform bone window

F) 3-year follow-up, with no complications such as facial paralysis, infection, relapse or spread.

\section{FIGURE 2:}

1. First histopathologic exam with diagnostic of "Suggestive of Paraganglioma" showing, a mass consisting of strands of rounded cells, with eosinophilic cytoplasm, oval nuclei and some areas of hemorrhage and necrosis.

2. CD68 positive marker showing macrophage activity and some interpose multinucleated giant cells, in the first biopsy.

3. H3.3G34W negative marker showing no activity for GCTB 4 years ago.

FIGURE 3: New histopathological features of the surgical specimen 4 years after. This tumor was characterized by proliferation of vascular spaces and fibroblastic cells with discrete collagen fibers and areas of hemorrhage surrounded by a large number of multinucleated giant cells, some with disorganized nuclei (A, B, C and D - asterisk ), others similar to Touton giant cells (E - arrow ), and different areas with osteoid material (arrow ). A large proliferation of xanthomatous-looking cells was also observed (F ), positive for periodic acid-Schiff staining (PAS, x200). 


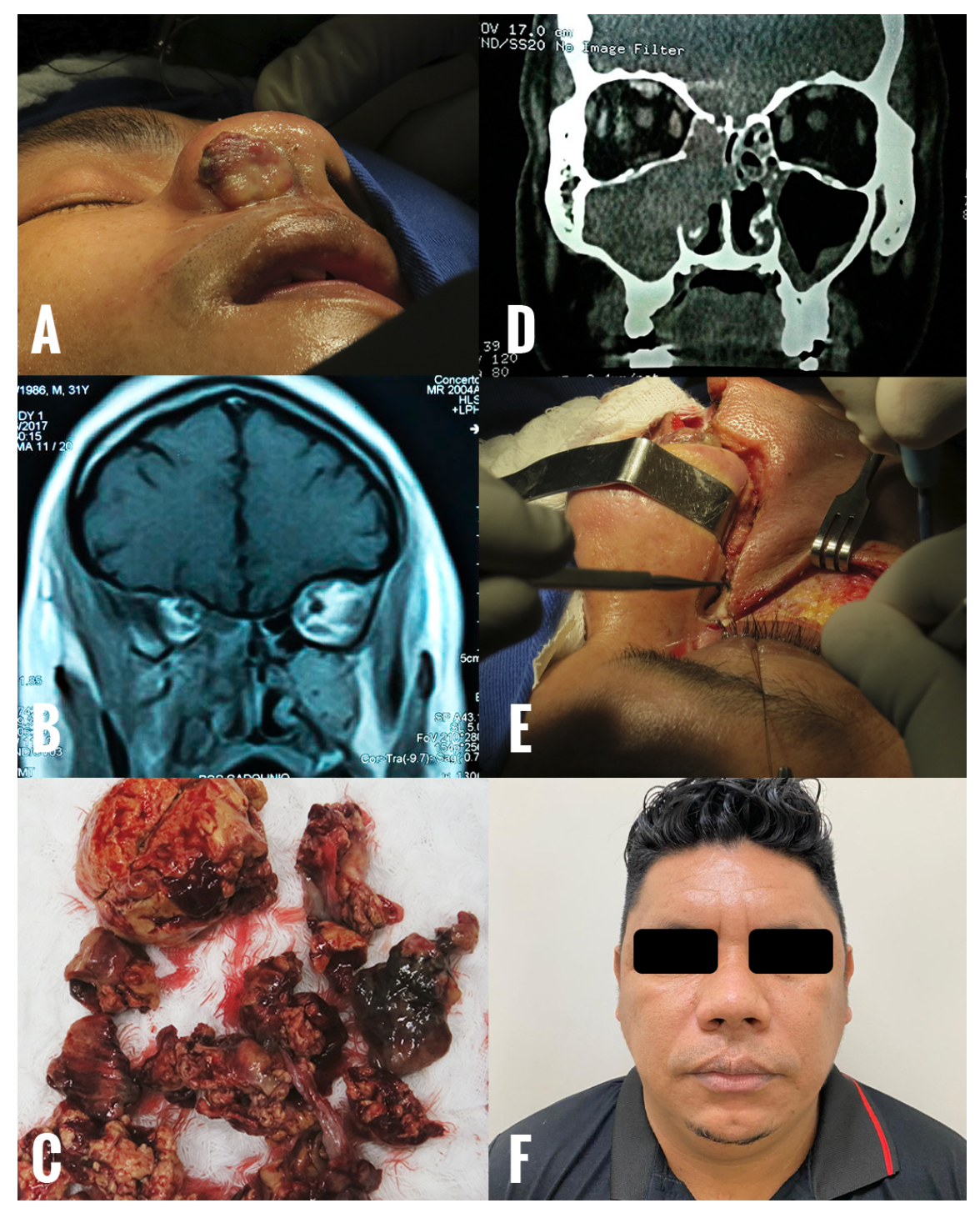




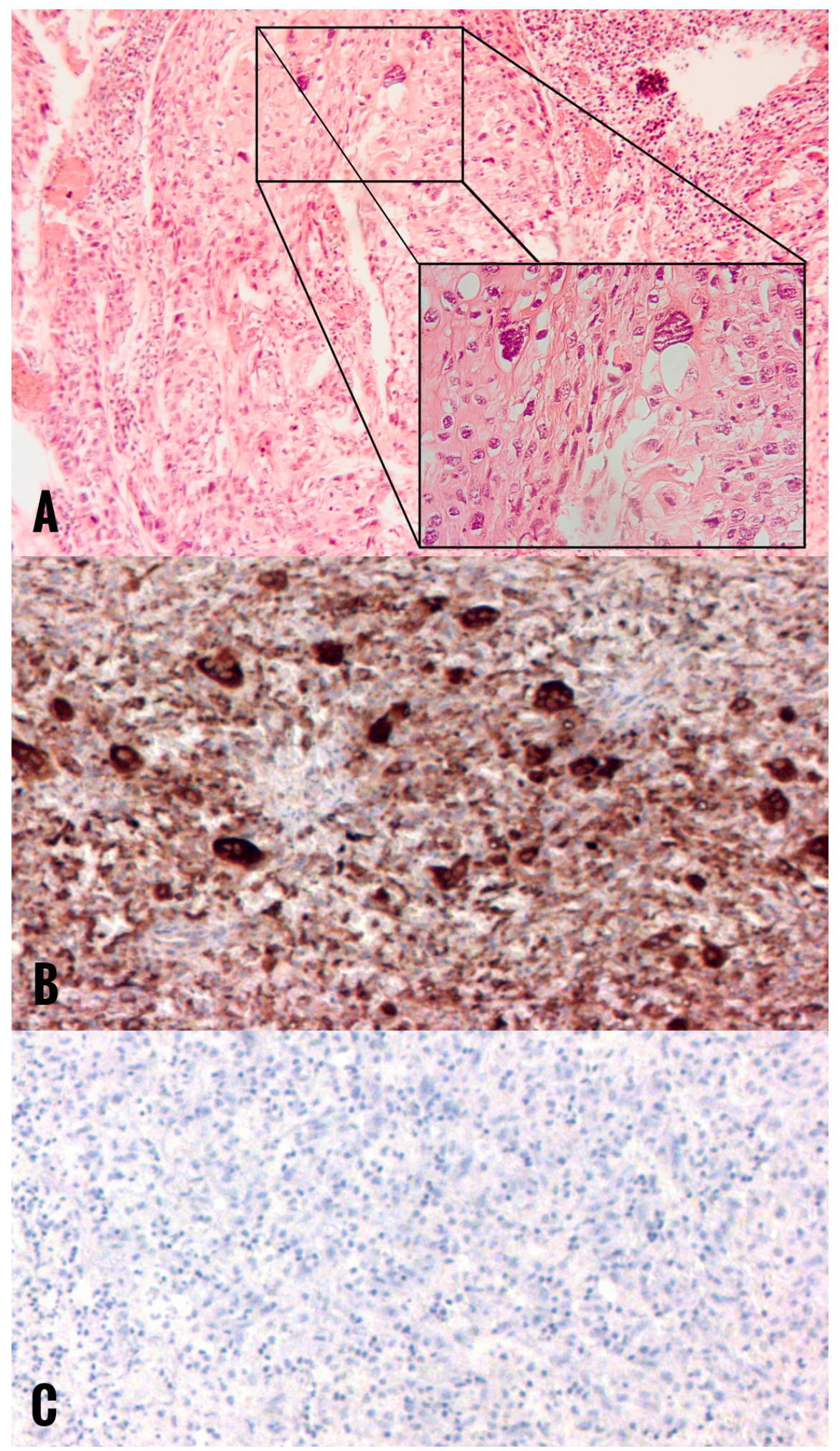




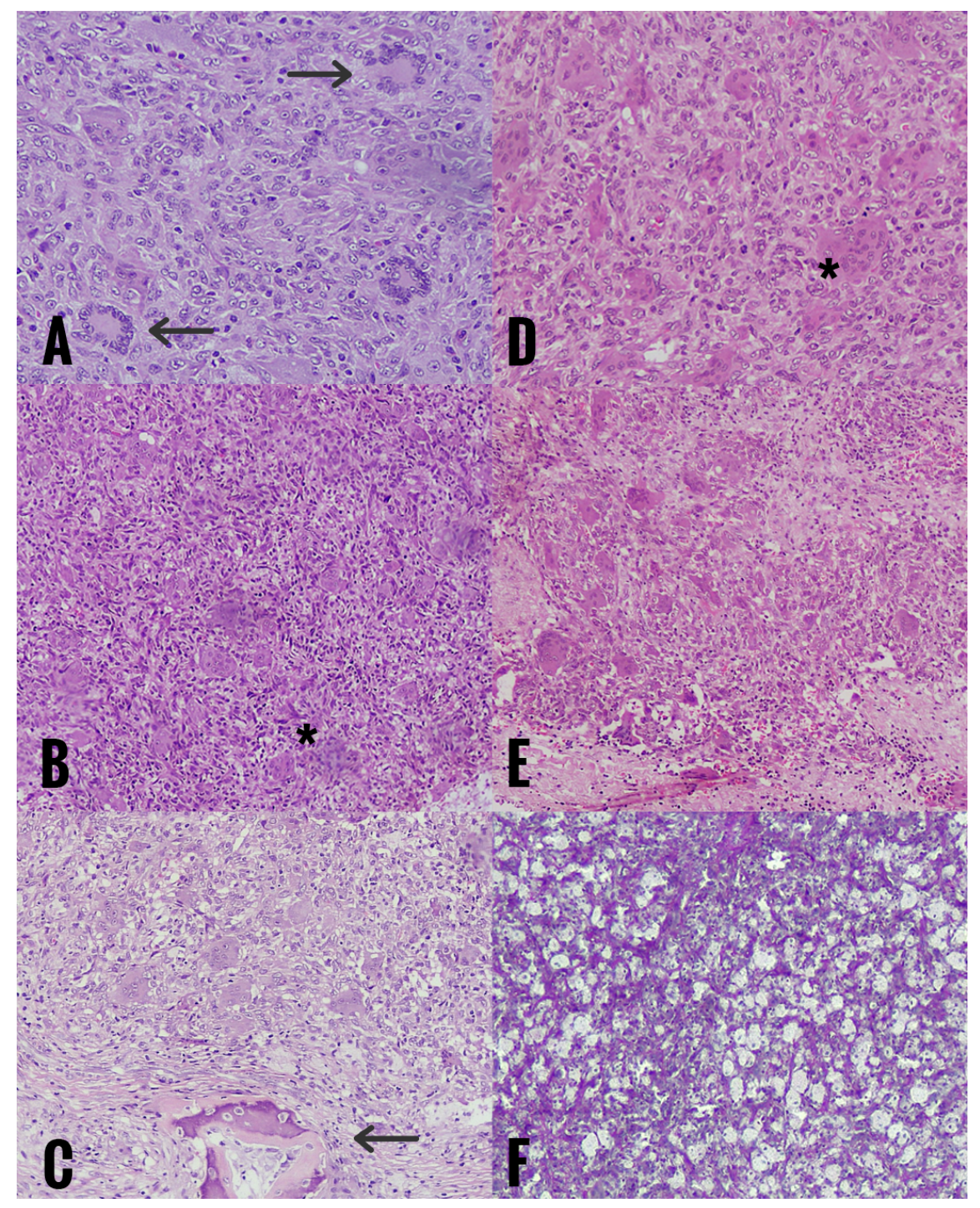

